\title{
Ten States of Nonvolatile Memory through Engineering Ferromagnetic Remanent Magnetization
}

\author{
Hai Zhong, Yan Wen, Yuelei Zhao, Qiang Zhang, Qikun Huang, Yanxue Chen, \\ Jianwang Cai, Xixiang Zhang, Run-wei Li, Lihui Bai, Shishou Kang, Shishen Yan,* \\ and Yufeng Tian*
}

Emerging nonvolatile multilevel memory devices have been regarded as a promising solution to meet the increasing demand of high-density memory with low-power consumption. In particular, decimal system of the new computers instead of binary system could be developed if ten nonvolatile states are realized. Here, a general remanent magnetism engineering method is proposed for realizing multiple reliable magnetic and resistance states, not depending on a specific material or device structure. Especially, as a proof-of-concept demonstration, ten states of nonvolatile memory based on the manipulation of ferromagnetic remanent magnetization have been revealed in both $\mathrm{Co} / \mathrm{Pt}$ magnetic multilayers with strong perpendicular magnetic anisotropy and MgO-based magnetic tunneling junctions at room temperature. Considering ferromagnets have been one of the key factors that enabled the information revolution from its inception, this state-of-the-art remanent magnetism engineering approach has a very broad application prospect in the field of spintronics.

\section{Introduction}

To meet the increasing demand of consumer electronics and multimedia storages, the realization of nonvolatile high-density memory and storage devices with low-power consumption is highly desired. ${ }^{[1-3]}$ Largely because of the simplicity to create dual stable magnetic or resistance states in the hardware, modern information technology is based on the binary logic. However, with the rapid development of thin film deposition and nanoscale device fabrication technologies, emerging nonvolatile memory devices based on the operation of multilevel states have attracted increasing attention. ${ }^{[4-7]}$ The challenge is how to achieve multilevel nonvolatile states that could be conveniently detected by magnetic and electrical

H. Zhong, Q. K. Huang, Prof. Y. X. Chen, Prof. L. H. Bai,

Prof. S. S. Kang, Prof. S. S. Yan, Dr. Y. F. Tian

School of Physics

State Key Laboratory of Crystal Materials

Shandong University

Jinan 250100, China

E-mail: shishenyan@sdu.edu.cn; yftian@sdu.edu.cn

H. Zhong, Y. Wen, Y. L. Zhao, Q. Zhang, Prof. X. X. Zhang

Physical Science and Engineering (PSE)

King Abdullah University of Science and Technology (KAUST)

Thuwal 239955, Kingdom of Saudi Arabia

Prof. J. W. Cai

Beijing National Laboratory for Condensed Matter Physics Institute of Physics

Chinese Academy of Sciences

Beijing 100190, China

Prof. R.-W. Li

Key Laboratory of Magnetic Materials and Devices

Ningbo Institute of Materials Technology and Engineering

Chinese Academy of Sciences

Ningbo 315201, China

Prof. S. S. Yan

Spintronics Institute

University of Jinan

Jinan 250022, China

The ORCID identification number(s) for the author(s) of this article can be found under https://doi.org/10.1002/adfm.201806460.

DOI: $10.1002 / \mathrm{adfm} .201806460$ transport measurements. In particular, decimal system of the new computers instead of binary system could be developed if ten nonvolatile states are realized, which would not only remarkably increase the memory density but also advance the development of neural networks, artificial intelligence and brain-like computing.

Recent research efforts toward multilevel nonvolatile devices include the phase change memory, ${ }^{\left[{ }^{[, 9]}\right.}$ resistive random access memory ${ }^{[10-12]}$ and ferroelectric transistor, ${ }^{[13-15]}$ where multiple resistance states are respectively demonstrated by utilizing phase change, resistance switching and multiferroic exchange coupling. In spite of intense research efforts and significant success, it is still very challenging to increase the number of levels with high fidelity more than four states. Moreover, it even seems impossible to achieve a common approach toward multiple resistance states in different systems, considering the fact that the dominated physical mechanisms vary from system to system. ${ }^{[8-15]}$ To overcome this issue, we propose a general remanent magnetism engineering method for realizing multiple reliable magnetic and resistance states, not depending on a specific material or device structure. It is well known that ferromagnetism and its related technology have been inextricably linked for centuries-from the ancient navigation compass, to modern magnetic reading heads, to present magnetoresistive random access memories. Ferromagnets have been one of the key factors that enabled the information revolution from its 

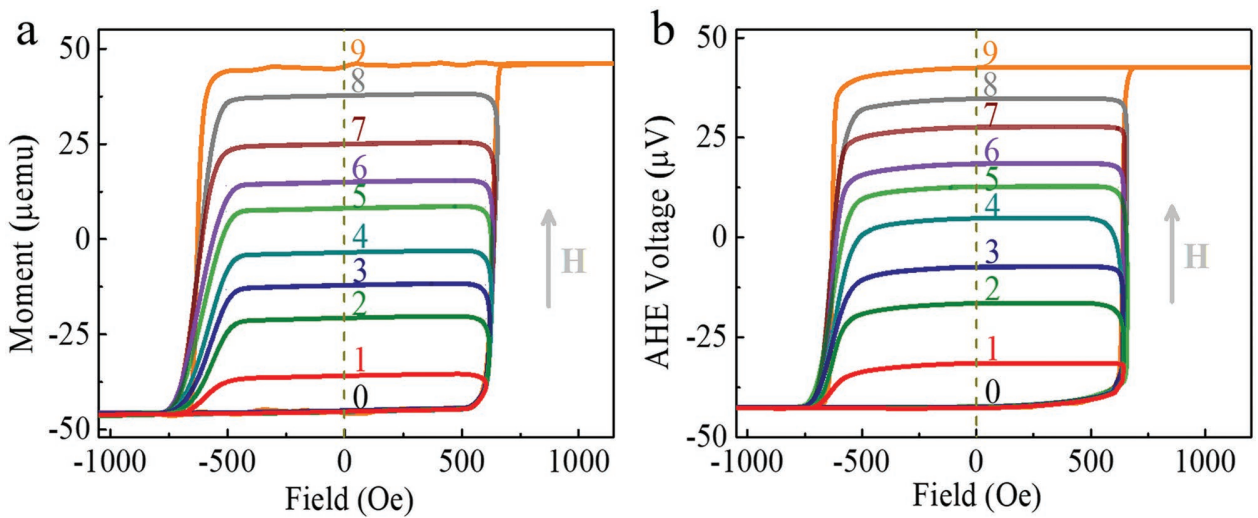

Figure 1. a) Room temperature minor magnetic hysteresis loops of [Co0.5/Pt0.3 $]_{4}$ multilayers measured with applied magnetic field perpendicular to the film plane. Ten remanent magnetization states are represented as 0-9. b) Anomalous Hall voltage as the "reading" of different remanent magnetization states with increasing "writing" magnetic field.

inception. Hence, this state-of-the-art remanent magnetization engineering approach has a very broad application prospect in the field of spintronics.

As a proof-of-concept demonstration, ten states of nonvolatile memory based on the manipulation of ferromagnetic remanent magnetization have been revealed in both magnetic thin film and magnetic tunneling junction (MTJ). In the cobalt/platinum $(\mathrm{Co} / \mathrm{Pt})$ multilayers, the detection of ten states of nonvolatile memory is demonstrated by conventional remanent magnetization, magneto-optical, and anomalous Hall effect measurements. Moreover, when the ferromagnets are incorporated into a functional MTJ, the ten states of nonvolatile memory can be read out by tunneling magnetoresistance (TMR) measurements.

\section{Results and Discussion}

\subsection{Ten Nonvolatile States in Magnetic Thin Films}

To begin with, we prepared the $\mathrm{Co} / \mathrm{Pt}$ multilayers with strong perpendicular magnetic anisotropy, ${ }^{[16,17]}$ which are currently a promising candidate for the high-density nonvolatile memory devices. The magnetic "writing" process is achieved by setting the magnetic field to a large negative saturation magnetic field (-1500 Oe), which provides a unified starting point. Then, one-to-one input magnetic field and output remanent magnetization is achieved by monotonically increasing "writing" magnetic field in the range of 615-670 Oe. As shown in Figure 1a, ten stable remanent magnetization states are obtained, which can represent 0 to 9 decimal numbers. Moreover, anomalous Hall effect can be used as a more convenient electrical "reading" of the remanent magnetization states of the magnetic thin films because it is proportional to the magnetization of the ferromagnetic materials. ${ }^{[18]}$ As illustrated in Figure $1 \mathrm{~b}$, ten corresponding anomalous Hall voltages are clearly detected after similar magnetic "writing" process. In addition, both the remanent magnetization and anomalous Hall voltage of a particular state show a high stability and repeatability as shown in Figure S1 (Supporting Information). In details, both the remanent magnetization (Figure S1c, Supporting Information) and Hall voltage (Figure S1d, Supporting Information) remain almost constant over $1 \mathrm{~h}$, and no significant variation has been detected after repeated for five times for both the minor magnetic hysteresis loops (Figure S1e, Supporting Information) and anomalous Hall effect (Figure S1f, Supporting Information). Further improvement of device reliability could be achieved by material optimization, such as using magnetic materials whose remanent magnetization changes more smoothly and linearly with external magnetic field.

It is worth noting that this common, simple and reliable manipulation method is only applicable to ferromagnet with multidomain states. Fortunately, multidomain states are energetically favorable to reduce the magnetostatic energy of a magnetic system through the formation of complex magnetic domains. ${ }^{[19,20]}$ Representative selections of magnetic domain images for a reference $\mathrm{Co} / \mathrm{Pt}$ multilayer at ten remanent magnetization states are shown in Figure 2, which are marked from 0 to 9 with increasing "writing" magnetic field. The bright and dark regions correspond to out-of-plane magnetization pointing down and up respectively. The most important feature here is the evolution of remanent magnetic domain states with increasing "writing" magnetic field, i.e., the dark regions monotonously increases with increasing "writing" magnetic field. Hence, by controlling the multidomain states through external stimuli, different remanent magnetization states are achieved, ${ }^{[20,21]}$ which eventually lead to the occurrence of multilevel states in the functional materials and devices. In addition, multidomain magnets could exhibit a much faster magnetization switching by virtue domain wall motion compared to single-domain magnets, allowing high-speed operation. ${ }^{[22]}$

\subsection{Ten Nonvolatile States in Magnetic Tunneling Junctions}

In the following, it is demonstrated that this powerful remanent magnetism engineering method can be adapted to functional magnetic heterojunctions to realize ten resistance states. For this illustration, IrMn/CoFe/ $\mathrm{Ru} / \mathrm{CoFeB} / \mathrm{MgO} / \mathrm{CoFeB} \mathrm{MTJ}$ is chosen because of its large tunneling magnetoresistance, which makes it one of the most important building blocks for spintronic devices. ${ }^{[23-25]}$ Here, synthetic antiferromagnetic pinned bottom layers are prepared by using $\mathrm{IrMn} / \mathrm{CoFe} / \mathrm{Ru} / \mathrm{CoFeB}$. 


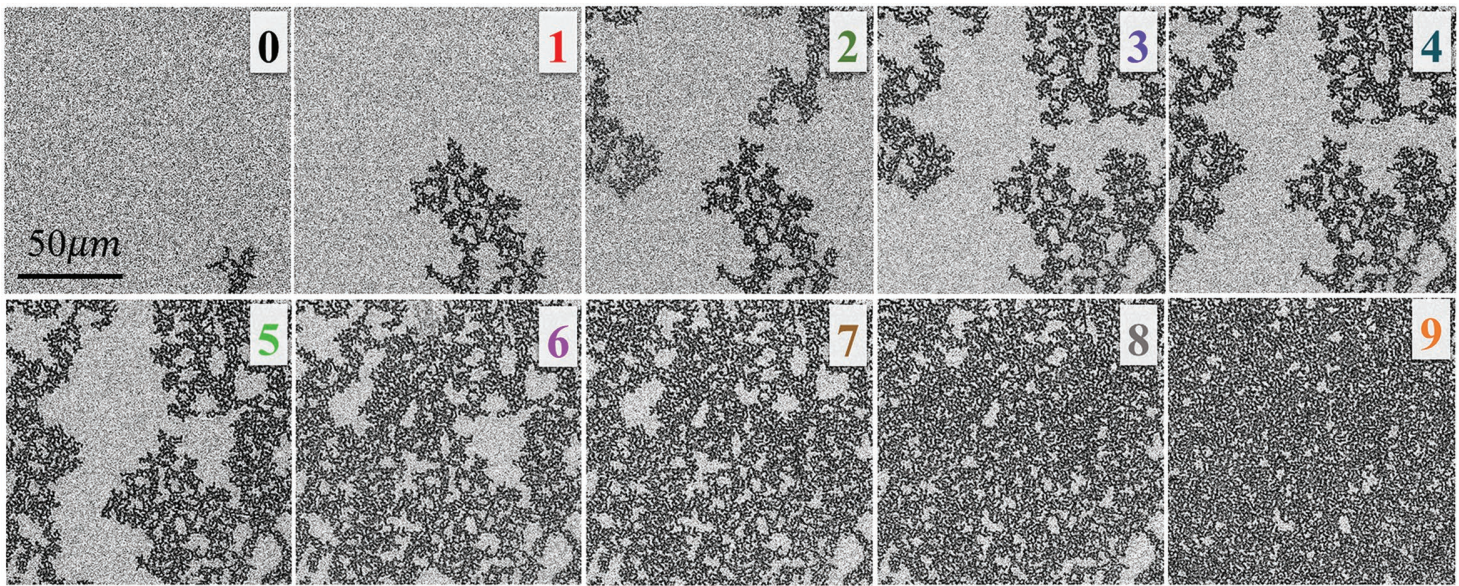

Figure 2. Different remanent MOKE images of the $[\mathrm{Co} 0.5 / \mathrm{Pt} 0.3]_{15}$ thin films with increasing "writing" magnetic field. The bright and dark regions correspond to out-of-plane magnetization pointing down and up, respectively.

And the thickness of $\mathrm{MgO}$ is $1 \mathrm{~nm}$ for large magnetoresistance. A stray field of 58 Oe is detected in Figure 3a. In this case, the remanent magnetization states in the free magnetic layer are defined as the states at the stray field, which can be kept by an additional hard magnetic layer in practical applications. In order to repeatedly manipulate the remanent magnetization of the top CoFeB free layer, the initial "writing" magnetic field is -800 Oe to completely reverse the magnetization of the free layer. But the moment of pinned bottom CoFeB layer is unaffected (Figure S2, Supporting Information). Then, by controlling the "writing" magnetic field, 10 remanent magnetization states are demonstrated (Figure 3a). It is believed that with increasing "writing" magnetic field, more and more magnetic moments of the free layer tend to align with the "writing" field, which is antiparallel to magnetic moments of the pinned CoFeB layers. In other words, the proportion of antiparallel alignment between free $\mathrm{CoFeB}$ and pinned $\mathrm{CoFeB}$ is increased. In an empirical model, the total junction resistance could be regarded as a parallel circuit, which consists of small resistance regions with the moments of two $\mathrm{CoFeB}$ sublayers parallel to each other and large resistance regions with the moments of two CoFeB sublayers antiparallel to each other. As a consequence, increasing the ratio of large resistance regions by controlling

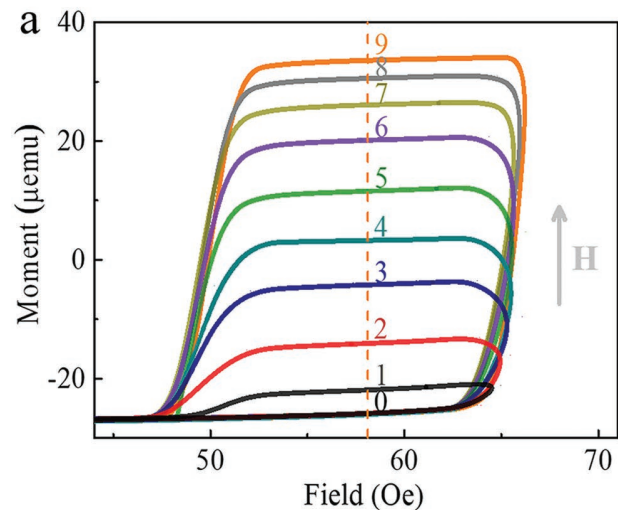

the domain states in the free layer while keeping the magnetic moments of pinned layer fixed leads to controllable junction resistance and TMR as well (Figure $3 \mathrm{~b}$ ). In addition to the tunable TMR, a clear stray field of 65 Oe is also detected. The slight difference in the shape of curves and the stray fields obtained from magnetic (58 Oe) and transport (65 Oe) measurements is due to the different sample size used during different measurements. The typical sample size used for the magnetic measurements is $5 \mathrm{~mm} \times 5 \mathrm{~mm}$, which is much larger than the area of MTJ (6 $\mu \mathrm{m}$ in diameter).

\subsection{MR Manipulation by Controlling the Antiferromagnetic Domain States}

Instead of controlling the domains of free layer, manipulating the remanent domain states of the pinned CoFeB layer by controlling the antiferromagnetic domain states should have the same function and better stability. To demonstrate this, we choose the MgO-based MTJ with a core structure of $\mathrm{NiFe/}$ $\mathrm{IrMn} / \mathrm{CoFeB} / \mathrm{MgO} / \mathrm{CoFeB}$. The direct contact between antiferromagnetic IrMn and $\mathrm{CoFeB}$ favors the strong exchange coupling between them. As a consequence, at the remanent states

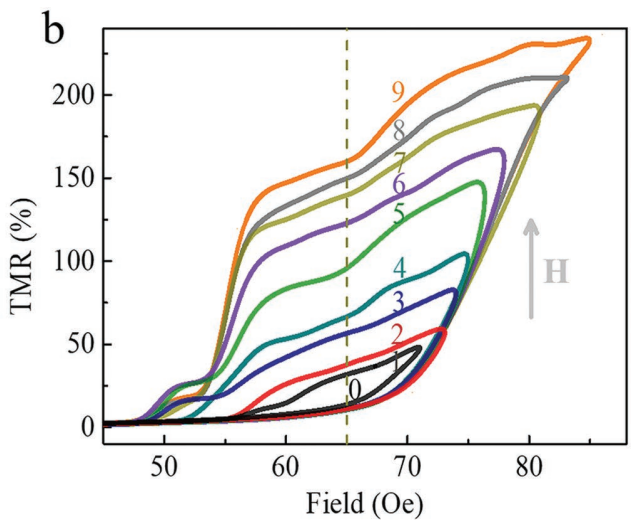

Figure 3. a) Room temperature minor magnetic hysteresis loops of CoFeB free layer in the synthetic antiferromagnetic pinned MgO-based MTJ. b) The corresponding TMR as the "reading" of different remanent magnetization states of the free layer. 


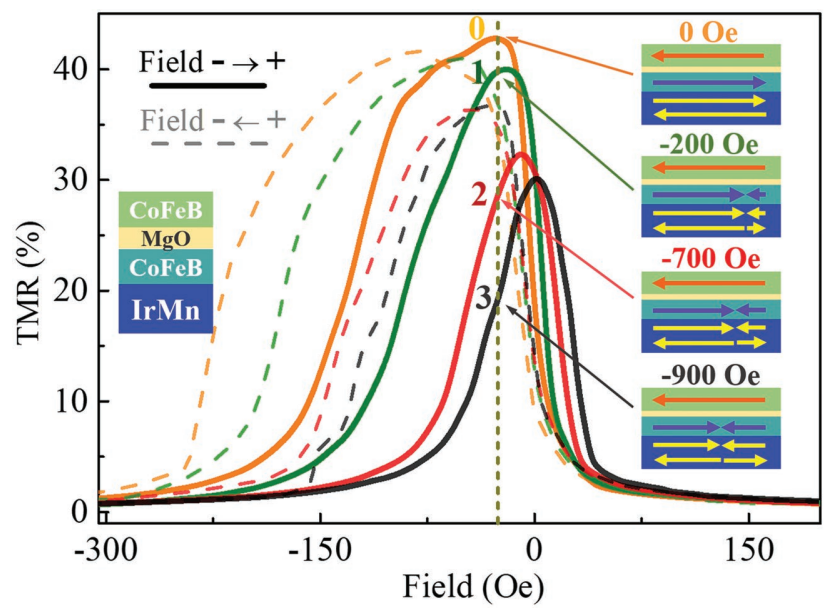

Figure 4. Room temperature TMR of direct pinned MgO-based MTJ measured after different field annealing process.

the magnetic moments of each domain in the bottom CoFeB are parallel to that of IrMn interface. Hence, the magnetic states of bottom $\mathrm{CoFeB}$ could be manipulated by controlling the multidomain states of the antiferromagnetic IrMn layer.

Experimentally, to realize a stable antiferromagnetic order in the IrMn layer which is grown on the ferromagnetic NiFe buffer layer, an initial induction field of +500 Oe is applied during the sample preparation, and then samples are annealed at $450{ }^{\circ} \mathrm{C}$ for an hour under zero magnetic field. After these operation, the whole antiferromagnetic IrMn layer can be approximately regarded as a single antiferromagnetic domain at room temperature, as schematically shown in the right insets of the 0 field case in Figure 4. Correspondingly, both the pinned CoFeB layer and the free CoFeB layer shows a single ferromagnetic domain, and their magnetization directions are antiparallel to each other at the MR peak position in Figure 4, which is labeled as 0.

In order to achieve remarkable manipulation of the multidomain states in IrMn layer, different field annealing processes have been further performed with the applied magnetic fields $(-200,-700$, and -900 Oe) opposite to the initial induction field of +500 Oe. In addition, the field annealing temperature is reduced to $440{ }^{\circ} \mathrm{C}$ to minimize the influence of microstructure variation on the MR, including crystallization, ion diffusion and surface roughness variation. With increasing the opposite annealing magnetic field, more and more magnetic domains in IrMn layer are reversed to the opposite direction of the initial configuration, which changes the single antiferromagnetic domain into multidomain states. Correspondingly, different magnetic states of bottom CoFeB could be manipulated by controlling the multidomain states of the antiferromagnetic IrMn layer. As an example, three additional MR states are obtained after the annealing in the magnetic fields of $-200,-700$, and -900 Oe, which are labeled as 1, 2, and 3 in Figure 4. It is clear that the peak MR value becomes small and the peak MR position moves to the right as the annealing field varies from 0 to -900 Oe. Their multidomain states at the MR peak are schematically shown in the right insets in Figure 4. In principle, more stable states could be realized by further optimize both the annealing temperature and the applied magnetic field during annealing. For simplicity and practical application, we can "write" and "read" the states of $0,1,2,3 \ldots . .$. and 9 at a fixed small bias magnetic field as marked in Figure 4 (which can be supplied by an additional hard magnetic layer in practical applications), where the free magnetic layer is in a single domain state and the fixed magnetic layer lies in multidomain states. It is also worth noting that MR curves shown in Figure 4 do not have a very sharp step-like switching, which means that the quality of synthesized MTJ needs to be further improved before it is suitable for realistic applications. It also suggests that actual domain structures could be much more complex than the schematic situation described above. For instance, a part of the domains of the pinned $\mathrm{CoFeB}$ could be decoupled from that of the antiferromagnetic IrMn. Though further in-depth investigation regarding the actual domain states is highly desired, the basic principle of the MR manipulation by controlling the antiferromagnetic domain is feasible. The key point is that the multidomain states of the pinned sublayer are controlled through the modulation of antiferromagnetic domain states, while the free sublayer is in a single domain state at the fixed small bias field.

Besides the clear manipulation of TMR, the asymmetry between increasing and decreasing branch of magnetic field reveals the existence of exchange bias effect, ${ }^{[26]}$ which is consistent with the strong exchange coupling between IrMn and bottom CoFeB. Also, advanced local- and fast-annealing technology, such as laser annealing, may be incorporated into functional devices to realize more efficient domain states manipulation in the future, which opens an alternative way toward multilevel nonvolatile memory devices.

\section{Conclusion}

In summary, we have experimentally demonstrated ten nonvolatile states in both $\mathrm{Co} / \mathrm{Pt}$ magnetic thin films and $\mathrm{MgO}$ based magnetic tunneling junctions at room temperature. This unique approach is based on the manipulation of remanent magnetism of a multidomain ferromagnet, which is not depending on a specific material or device structure. Easy to operate along with tunable multiple magnetic and resistance states makes this remanent magnetism engineering approach an attractive way toward multilevel nonvolatile memory devices, which is of particular interest due to their considerable highdensity memory capacities. Moreover, this state-of-the-art technique has a very broad application prospect, considering the fact that ferromagnetic materials have already been widely used in many aspects of both industrial production and daily life.

\section{Experimental Section}

Preparation of Synthetic Antiferromagnetic Pinned MgO Magnetic Tunneling Junctions: The nominal structure of the synthetic antiferromagnetic pinned $\mathrm{MgO} \mathrm{MT}$ s is $\mathrm{Ta}(3) / \mathrm{CuN}(10) / \mathrm{Ta}(5) / \mathrm{Ru}(5) /$ $\mathrm{Ir}_{20} \mathrm{Mn}_{80}(8) / \mathrm{Co}_{70} \mathrm{Fe}_{30}(2.5) / \mathrm{Ru}(0.85) / \mathrm{Co}_{40} \mathrm{Fe}_{40} \mathrm{~B}_{20}$ (3.0)/MgO (1.0)/ $\mathrm{Co}_{40} \mathrm{Fe}_{40} \mathrm{~B}_{20}(2.5) / \mathrm{Ta}(8) / \mathrm{Cu}(10) / \mathrm{Ru}(5)$. The numbers in parenthesis stand for thickness in $\mathrm{nm}$. This group of samples was deposited on Si (001) substrates coated with $300 \mathrm{~nm}$ thick $\mathrm{SiO}_{2}\left(\mathrm{Si}_{-} \mathrm{SiO}_{2}\right)$ by using Singulus ROTARIS physical vapor deposition (PVD) system at room temperature. After deposition, samples were annealed at $360^{\circ} \mathrm{C}$ for $2 \mathrm{~h}$ with an in-plane magnetic field of 0.8 Tesla. Finally, circular-shape MT)s with a 
diameter $6 \mu \mathrm{m}$ were fabricated by using standard optical lithography, reactive ion etching, and lift-off technology.

Preparation of Direct Antiferromagnetic Pinned MgO Magnetic Tunneling Junctions: The nominal structure of the direct antiferromagnetic pinned $\mathrm{MgO} \mathrm{MT}$ ss is $\mathrm{Ta}(10) / \mathrm{Cu}(5) / \mathrm{Ta}(10) / \mathrm{Ni}_{81} \mathrm{Fe}_{19}(5) / \mathrm{Ir}_{20} \mathrm{Mn}_{80}(8) /$ $\mathrm{CO}_{40} \mathrm{Fe}_{40} \mathrm{~B}_{20}(4.0) / \mathrm{MgO}(1.6) / \mathrm{Co}_{40} \mathrm{Fe}_{40} \mathrm{~B}_{20}(3.0) / \mathrm{Ta}(10) / \mathrm{Au}(10)$. This group of samples was deposited on (001) $\mathrm{Si}_{-} \mathrm{SiO}_{2}$ substrates by homemade magnetron sputtering at room temperature, where the base pressure is better than $5 \times 10^{-5} \mathrm{~Pa}$. During the deposition, an in-plane magnetic field of 500 Oe was applied to induce exchange bias, and the direction of this initial induction field was defined as the positive direction. Then, square-shaped crossbar MTJs with an effective area of $5 \mu \mathrm{m} \times 5 \mu \mathrm{m}$ were fabricated by using optical lithography and Ar-ion milling technology. In order to demonstrate the MR manipulation by controlling the antiferromagnetic domain states, the studied samples were first annealed at $450{ }^{\circ} \mathrm{C}$ for $1 \mathrm{~h}$ under zero magnetic field, and then annealed at $440{ }^{\circ} \mathrm{C}$ for $1 \mathrm{~h}$ with $-200,-700$, and -900 Oe magnetic field respectively.

Preparation of Co/Pt Multilayers: The $\mathrm{Ta}(1) / \mathrm{Pt}(6) /[\mathrm{Pt} 0.3 / \mathrm{Co} 0.5]_{n} / \mathrm{Ta}$ (3) multilayers were also deposited on the (001) $\mathrm{Si}_{-} \mathrm{SiO}_{2}$ by our homemade magnetron sputtering at room temperature. The growth rate of $\mathrm{Pt}$ and Co was 0.0556 and $0.1923 \AA \mathrm{s}^{-1}$, respectively. For the anomalous Hall measurements, without specification, samples were patterned into Hall bar structure with a channel $10 \mu \mathrm{m}$ in width and $70 \mu \mathrm{m}$ in length by using optical lithography and Ar-ion milling. In order to improve the signal-to-noise ratio, the period of $[\mathrm{Co} / \mathrm{Pt}]_{n}$ was increased from 4 to 15 for the magneto-optical Kerr measurements.

Magnetic Properties' Characterization: The magnetic properties were measured by using superconducting quantum interference devices (SQUIDs, Quantum Design, MPMS-XL 7). The typical sample size was $5 \mathrm{~mm} \times 5 \mathrm{~mm}$. As shown in Figures $1 \mathrm{a}$ and $3 \mathrm{a}$, the magnetic field was swept from negative saturation magnetic field to different positive magnetic field and then swept back to negative saturation field for each minor hysteresis loop measurements.

Magnetic Domain Image Characterization: The magnetic domain images of $[\mathrm{Co} / \mathrm{Pt}]_{15}$ samples were obtained with a magneto-optical Kerr-microscope manufactured by Evico magnetics $\mathrm{GmbH}$. During the measurements, the sample was first saturated by a magnetic field of $-1000 \mathrm{Oe}$, and then MOKE images at different remanent magnetization states were captured after monotonically increasing the "writing" magnetic field.

Electrical Transport Measurements: Keithley 2400 current source meter and Keithley $2182 \mathrm{~A}$ voltage meter were used for the anomalous Hall effect and MR characterization. Four point measurements were used during the MR measurements. The enhanced TMR signal in the synthetic antiferromagnetic pinned MgO MTJs was attributed to the geometric effects. ${ }^{[27,28]}$

\section{Supporting Information}

Supporting Information is available from the Wiley Online Library or from the author.

\section{Acknowledgements}

This work was supported by the National Science Foundation of China (Grant Nos. 11434006, 11774199, and 51871112), the National Basic Research Program of China (Grant No. 2015CB921502), the 111 ProjectB13029, and the Taishan Scholar Program of Shandong Province. H.Z. was supported by the King Abdullah University of Science and Technology (KAUST) during his visit to KAUST. X.X.Z. acknowledges the support from KAUST.

\section{Conflict of Interest}

The authors declare no conflict of interest.

\section{Keywords}

magnetic tunneling junctions, magnetoresistance, multilevel states, nonvolatile memory, remanent magnetization

Received: September 12, 2018

Revised: October 24, 2018

Published online:

[1] S. A. Wolf, D. D. Awschalom, R. A. Buhrman, J. M. Daughton, S. von Molnar, M. L. Roukes, A. Y. Chtchelkanova, D. M. Treger, Science 2001, 294, 1488.

[2] I. Žutić, J. Fabian, S. Das Sarma, Rev. Mod. Phys. 2004, 76, 323.

[3] S. D. Bader, S. S. P. Parkin, Annu. Rev. Condens. Matter Phys. 2010, $1,71$.

[4] Y. L. Guo, C.-A. Di, S. H. Ye, X. N. Sun, J. Zheng, Y. G. Wen, W. P. Wu, G. Yu, Y. Q. Liu, Adv. Mater. 2009, 21, 1954.

[5] C. Moreno, C. Munuera, S. Valencia, F. Kronast, X. Obradors, C. Ocal, Nano Lett. 2010, 10, 3828.

[6] D. Lee, S. M. Yang, T. H. Kim, B. C. Jeon, Y. S. Kim, J.-G. Yoon, H. N. Lee, S. H. Baek, C. B. Eom, T. W. Noh, Adv. Mater. 2012, 24, 402.

[7] M. J. Rozenberg, I. H. Inoue, M. J. Sanchez, Phys. Rev. Lett. 2004, 92, 178302.

[8] H.-S. P. Wong, S. Raous, S. Kim, J. L. Liang, J. P. Reifenberg, B. Rajendran, M. Asheghi, K. E. Goodson, Proc. IEEE 2010, 98, 2201.

[9] M. Wuttig, N. Yamada, Nat. Mater. 2007, 6, 824.

[10] W. M. Lü, C. J. Li, L. M. Zheng, J. X. Xiao, W. N. Lin, Q. Li, X. R. Wang, Z. Huang, S. W. Zeng, K. Han, W. X. Zhou, K. Y. Zeng, J. S. Chen, Ariando, W. W. Cao, T. Venkatesan, Adv. Mater. 2017, 29, 1606165.

[11] R. Waser, M. Aono, Nat. Mater. 2007, 6, 833.

[12] S. Balatti, S. Larentis, D. C. Gilmer, D. Ielmini, Adv. Mater. 2013, 25, 1474.

[13] A. G. Boni, C. Chirila, I. Pasuk, R. Negrea, I. Pintilie, L. Pintilie, Phys. Rev. Appl. 2017, 8, 034035

[14] X. Y. Zhao, J. H. Wen, B. Yang, H. C. Zhu, Q. Q. Cao, D. H. Wang, Z. H. Qian, Y. W. Du, ACS Appl. Mater. Interfaces 2017, 9, 36038.

[15] W. J. Hu, Z. H. Wang, W. L. Yu, T. Wu, Nat. Commun. 2016, 7, 10808.

[16] R. Sbiaa, H. Meng, S. N. Piramanayagam, Phys. Status Solidi RRL 2011, 5, 413.

[17] B. Dieny, M. Chshiev, Rev. Mod. Phys. 2017, 89, 025008.

[18] N. Nagaosa, J. Sinova, S. Onoda, A. H. MacDonald, N. P. Ong, Rev. Mod. Phys. 2010, 82, 1539.

[19] J. M. D. Coey, Magnetism and Magnetic Materials, Cambridge University Press, Cambridge, UK 2010.

[20] K. F. Huang, D. S. Wang, M. H. Tsai, H. H. Lin, C. H. Lai, Adv. Mater. 2017, 29, 1601575.

[21] S. S. P. Parkin, M. Hayashi, L. Thomas, Science 2008, 320, 190.

[22] J.-M. Hu, Z. Li, L.-Q. Chen, C. W. Nan, Adv. Mater. 2012, 24, 2869.

[23] S. Ikeda, J. Hayakawa, Y. Ashizawa, Y. M. Lee, K. Miura, H. Hasegawa, M. Tsunoda, F. Matsukura, H. Ohno, Appl. Phys. Lett. 2008, 93, 082508.

[24] S. Yuasa, T. Nagahama, A. Fukushima, Y. Suzuki, K. Ando, Nat. Mater. 2004, 3, 868 .

[25] S. S. P. Parkin, C. Kaiser, A. Panchula, P. M. Rice, B. Hughes, M. Samant, S.-H. Yang, Nat. Mater. 2004, 3, 862.

[26] J. Nogues, I. K. Schuller, J. Magn. Magn. Mater. 1999, 192, 203.

[27] J. S. Moodera, L. R. Kinder, J. Nowak, P. LeClair, R. Meservey, Appl. Phys. Lett. 1996, 69, 708.

[28] R. J. M. van de Veerdonk, J. Nowak, R. Meservey, J. S. Moodera, W. J. M. de Jonge, Appl. Phys. Lett. 1997, 71, 2839. 